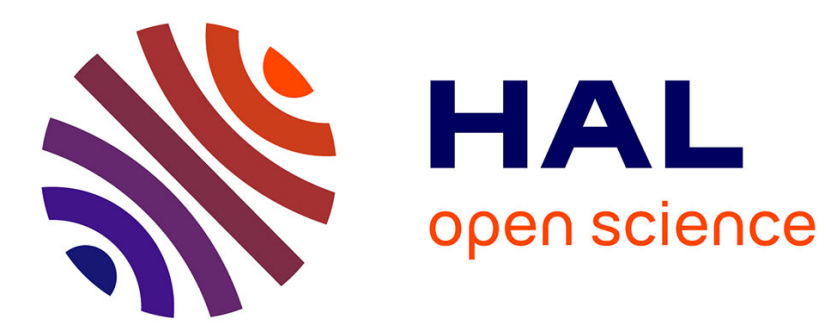

\title{
The Mayer and Minimum Time Problems with Stratified State Constraints *
}

C. Hermosilla, P.R. Wolenski, Hasnaa Zidani

\section{To cite this version:}

C. Hermosilla, P.R. Wolenski, Hasnaa Zidani. The Mayer and Minimum Time Problems with Stratified State Constraints *. Set-Valued and Variational Analysis, 2017, 10.1007/s11228-017-0413-z . hal01517510

\section{HAL Id: hal-01517510 https://hal-ensta-paris.archives-ouvertes.fr/hal-01517510}

Submitted on 9 May 2017

HAL is a multi-disciplinary open access archive for the deposit and dissemination of scientific research documents, whether they are published or not. The documents may come from teaching and research institutions in France or abroad, or from public or private research centers.
L'archive ouverte pluridisciplinaire HAL, est destinée au dépôt et à la diffusion de documents scientifiques de niveau recherche, publiés ou non, émanant des établissements d'enseignement et de recherche français ou étrangers, des laboratoires publics ou privés. 


\title{
The Mayer and Minimum Time Problems with Stratified State Constraints*
}

\author{
C. Hermosilla ${ }^{\dagger}$ \\ P. R. Wolenski ${ }^{\ddagger}$ \\ H. Zidani ${ }^{\S}$
}

April 4, 2017

\begin{abstract}
This paper studies optimal control problems with state constraints by imposing structural assumptions on the constraint domain coupled with a tangential restriction with the dynamics. These assumptions replace pointing or controllability assumptions that are common in the literature, and provide a framework under which feasible boundary trajectories can be analyzed directly. The value functions associated with the state constrained Mayer and minimal time problems are characterized as solutions to a pair of Hamilton-Jacobi inequalities with appropriate boundary conditions. The novel feature of these inequalities lies in the choice of the Hamiltonian.
\end{abstract}

Keywords. State constraints, stratified structure, Mayer problem, minimal time function, Hamilton-Jacobi equations.

\section{Introduction}

The formulation of realistic optimal control problems typically involves constraints on state variables that seek to model regions of the state space where perhaps the operation is unsafe or the dynamic model is no longer valid. The underlying dynamical system used throughout this paper is the differential inclusion

$$
\left\{\begin{array}{l}
\dot{y}(s) \in F(y(s)) \quad \text { a.e. } s \in[t, T] \\
y(t)=x
\end{array}\right.
$$

where $T \in \mathbb{R}$ is fixed and the dynamic data $F: \mathbb{R}^{N} \rightrightarrows \mathbb{R}^{N}$ is a multifunction satisfying standard hypotheses. We will be concerned when trajectories of (DI) also satisfy the state constraint

$$
y(s) \in \mathcal{K} \quad s \in[t, T]
$$

where the admissible state domain $\mathcal{K} \subseteq \mathbb{R}^{N}$ is a closed set with a special local structure. If $y(\cdot)$ is a solution satisfying $(\mathrm{DI})$ and $(\mathrm{SC})$, then we say $y(\cdot)$ is a solution of $(\mathrm{DI})_{\mathcal{K}}$. The initial data $t \in(-\infty, T]$ and $x \in \mathbb{R}^{N}$ are parameters, and a solution to (DI) is denoted by $y_{t, x}(\cdot)$. We are also given a terminal cost function $\psi: \mathbb{R}^{d} \rightarrow \mathbb{R} \cup\{+\infty\}$ that is assumed to be a l.s.c. (1.s.c.) function bounded below on bounded sets. The (state-constrained) Mayer problem is designated as $\mathcal{P}(t, x)$, and consists of

$$
\min \left\{\psi\left(y_{t, x}(T)\right) \mid y_{t, x}(\cdot) \text { satisfies }(\mathrm{DI})_{\mathcal{K}}\right\} \text {. }
$$

\footnotetext{
* This work was partially supported by iCODE institue, the European Union under the 7th Framework Programme FP7-PEOPLE-2010-ITN Grant agreement number 264735-SADCO and by the ANR project HJNet ANR-12-BS010008-01.

${ }^{\dagger}$ Departamento de Matemáticas, Universidad Técnica Federico Santa María, Valparaíso, Chile. cristopher.Hermosill@usm.cl

${ }^{\ddagger}$ Department of Mathematics, Louisiana State University, Baton Rouge, Louisiana, USA. wolenski@math.lsu.edu

$\S$ Unité des Mathématiques Appliquées (UMA), ENSTA ParisTech, Palaiseau, France.
} Hasnaa.Zidani@ensta-paristech.fr 
The optimal value in $(\mathcal{P}(t, x))$ is denoted by $\vartheta(t, x)$. Here and throughout the paper, the usual convention is that $\inf \emptyset=+\infty$ (and $\sup \emptyset=-\infty)$. Under our assumptions, the value function is also l.s.c. and therefore may exhibit discontinuities. Our main goal is to characterize the graph of $\vartheta(\cdot, \cdot)$ by means of a generalized Hamilton-Jacobi (HJ) equation. We mention that the epigraph of $\vartheta(\cdot, \cdot)$ has been characterized in $[1,16]$ that suggested and lead to numerical algorithms, but working directly with the graph is a somewhat more delicate task. In the case when $\mathcal{K}=\mathbb{R}^{d}$ and $\psi(\cdot)$ is Lipschitz continuous, it is known that the value function $\vartheta(\cdot, \cdot)$ is the unique continuous viscosity solution of a Hamilton-Jacobi equation $[14,6]$. This result is extended to the l.s.c. setting with bilateral equations in $[8,17]$.

In the presence of state constraints, it has been shown that the value function satisfies a stateconstrained HJ equation, which in our setting takes the form

$$
\begin{aligned}
-\partial_{t} \vartheta(t, x)+\mathcal{H}\left(x, \nabla_{x} \vartheta(t, x)\right) & \geq 0 \quad \forall(t, x) \in(-\infty, T) \times \mathcal{K}, \\
-\partial_{t} \vartheta(t, x)+\mathcal{H}\left(x, \nabla_{x} \vartheta(t, x)\right) & \leq 0 \quad \forall(t, x) \in(-\infty, T) \times \operatorname{int} \mathcal{K}, \\
\vartheta(T, x) & =\psi(x) \quad \forall x \in \mathcal{K},
\end{aligned}
$$

where $\mathcal{H}(x, p):=\max _{v \in F(x)}-\langle v, p\rangle$. The precise meaning of the derivatives occurring in (1.1a) and $(1.1 \mathrm{~b})$ is in the viscosity sense and will be reviewed in Section 2. The fact that the sub-solution inequality (1.1b) is to hold only in the interior of $\mathcal{K}$ leads to an under-determined system of equations which cannot assure uniqueness of the overall equation unless some additional properties linking the dynamic data and the constraint set $\mathcal{K}$ are satisfied; see $[11,21]$. The invoked linkage assumption investigated the most in the literature is called an "Inward Pointing Condition" (IPC), and was used in $[25,26]$ by Soner to investigate constrained viscosity solution of HJB equations. Loosely speaking, it says that at each point of the boundary of $\mathcal{K}$, there exists a field of the system pointing strictly inward to $\mathcal{K}$. In particular, the state space must have an interior whose closure is minimally the entire domain. Under (IPC) and when $\psi$ is Lipschitz continuous, the value function is also Lipschitz continuous in $\mathcal{K}$, and it can be established that it is the unique solution to (1.1). We refer to [23, 24] for further refinements of (IPC). Another condition investigated in the literature is the so-called "Outward Pointing Condition" (OPC). This condition ensures that at each point $x$ at the boundary of $\mathcal{K}$, there exists an admissible velocity pointing outside (which essentially means the point can be reached from the inside) [10]. Let us point out that IPC and OPC have also been used for other purposes in Optimal control (optimality conditions); see for instance [2, 3, 4, 15].

In the general case without any of the above-mentioned pointing qualifications, the value function fails to be the unique viscosity solution to the constrained HJ equation (1.1) because (1.1) does not explicitly describe the behavior of the value function along admissible trajectories evolving on the boundary for a nontrivial time interval, see [11].

In this paper, we follow the same line of research undertaken in [20] and derive a new characterization of the value function, for Mayer and minimum time problems, in term of unique Bilateral viscosity solution to an adequate Hamilton-Jacobi equation. The main feature of this paper is to assume that the state constraint set $\mathcal{K}$ is endowed with a stratified structure while the dynamic data $F$ satisfies standard assumptions of differential inclusion theory along with a structural tangential condition. In particular, the constraint set $\mathcal{K}$ may have empty interior or have subdomains of different dimension, conditions precluded by any of the pointed conditions. We will also require that on each stratified subdomain, either the admissible velocities throughout that subdomain form a Lipschitz multifunction, or there are no admissible velocities at all throughout the subdomain. Moreover, our proof techniques require a local controllability assumption in order to treat obstreperous feasible arcs that exhibit a chattering or Zeno-like behavior.

Let us mention that the Mayer problem considered here involves an end-point cost which by itself can be discontinuous, whereas in the infinite horizon problem in [20] the distributed cost is assumed to be Lipschitz continuous and there is no final cost. Moreover, in the present work, the approximate technique that will be used to handle chattering trajectories concerns directly the trajectories, while in [20] the approximation uses the structure of the control inputs. Another feature of the present work 
is that the dynamics $F$ is considered to be uppersemi-continuous on the set $\overline{\mathcal{K}}$ whereas in [20] it is assumed to be Lipschitz continuous.

The paper is organized as follows. In section 2, Mayer problem is introduced along with the assumptions. Then a characterisation of the value function is stated in Theorem 2.2. This result is discussed on a more specific example in subsection 2.4. Section 3 is devoted to the analysis of some increasing principles for stratified systems and gives a proof to the main result Theorem 2.2. Section 5 focused on the characterization for a state-constrained minimum time problem, and section 6 gives some comments on the main features of the paper. Finally, an appendix gathers some technical results that are needed in section 3 .

\section{Setting of the problem}

We lay out the assumptions of our problem in this section, give the main result and provide a few examples to illustrate its applicability.

\subsection{Notation}

Throughout this paper, $\langle\cdot, \cdot\rangle$ denotes the Euclidean inner product on $\mathbb{R}^{N}, \mathbb{B}$ the unit open ball $\{x \in$ $\left.\mathbb{R}^{N}:|x|<1\right\}$ and $\mathbb{B}(x, r)=x+r \mathbb{B}$. For any set $S \subseteq \mathbb{R}^{N}$, int(S), $\overline{\mathrm{S}}$, and $\partial S$ denote its interior, closure and boundary, respectively. Also for $S$ convex we denote by $r$-int $(S)$ and $r$-bdry $(S)$ its relative interior and boundary, respectively. The indicator $\mathbb{1}_{S}$ and the characteristic $\chi_{S}$ functions are respectively given by

$$
\mathbb{1}_{S}(x)=\left\{\begin{array}{ll}
1 & \text { if } x \in S \\
0 & \text { if not }
\end{array}, \quad \chi_{S}(x)=\left\{\begin{array}{ll}
0 & \text { if } x \in S \\
+\infty & \text { if not }
\end{array} .\right.\right.
$$

The distance function to $S$ is $\operatorname{dist}_{S}(x)=\inf \{|x-y|: y \in S\}$ and in the case the infimum is attained we call the set of solution the projections of $x$ over $S$ and we denote it by $\operatorname{proj}_{S}(x)$. Let $S_{1}$ and $S_{2}$ be two compact set, then the Hausdorff distance is given by

$$
d_{H}\left(S_{1}, S_{2}\right)=\max \left\{\sup _{x \in S_{2}} \operatorname{dist}_{S_{1}}(x), \sup _{x \in S_{1}} \operatorname{dist}_{S_{2}}(x)\right\} .
$$

We adopt the convention that $d_{H}(\emptyset, \emptyset)=0$ and $d_{H}(\emptyset, S)=+\infty$ if $S \neq \emptyset$. For a given function $\varphi: \mathbb{R}^{N} \rightarrow \mathbb{R} \cup\{+\infty\}$, the epigraph of this function is the set

$$
\text { epi } \varphi=\left\{(x, r) \in \mathbb{R}^{N} \times \mathbb{R} \mid r \geq \varphi(x)\right\} .
$$

The effective domain of $\varphi$ is given by dom $\varphi=\left\{x \in \mathbb{R}^{N} \mid \varphi(x) \in \mathbb{R}\right\}$. If $\Gamma$ is a set-valued map, then $\operatorname{dom} \Gamma$ is the set points for which $\Gamma(x) \neq \emptyset$.

For an embedded manifold of $\mathbb{R}^{N}$, the tangent space to $\mathcal{M}$ at $x$ is denoted by $\mathcal{T}_{\mathcal{M}}(x)$.

In all the paper, the abrreviations "l.s.c.", "u.s.c." and "w.r.t" stand respectively for "lower semicontinuous", "upper semicontinuous" and "with respect to".

\subsection{The Mayer problem and basic assumptions}

The class of control problems we are considering in this paper do not necessarily satisfy any qualification hypothesis. Here, we require two principal assumptions, $\left(H_{0}\right)$ and $\left(H_{1}\right)$ stated below. The first one is that the state-constraints set admits a sufficiently regular partition into smooth manifolds or strata. More precisely,

$$
\mathcal{K} \text { is a closed and stratifiable subset of } \mathbb{R}^{N},
$$

that is, there exists a locally finite collection $\left\{\mathcal{M}_{i}\right\}_{i \in \mathcal{I}}$ of embedded manifolds of $\mathbb{R}^{N}$ such that: 
- $\mathcal{K}=\bigcup_{i \in \mathcal{I}} \mathcal{M}_{i}$ and $\mathcal{M}_{i} \cap \mathcal{M}_{j}=\emptyset$ when $i \neq j$.

- If $\mathcal{M}_{i} \cap \overline{\mathcal{M}}_{j} \neq \emptyset$, necessarily $\mathcal{M}_{i} \subseteq \overline{\mathcal{M}}_{j}$ and $\operatorname{dim}\left(\mathcal{M}_{i}\right)<\operatorname{dim}\left(\mathcal{M}_{j}\right)$.

Remark 2.1 Notice that if $\operatorname{int}(\mathcal{K}) \neq \emptyset$ and $\partial \mathcal{K}$ is smooth, then a possible stratification satisfying $\left(H_{0}\right)$ is the one composed of only two strata, namely, $\mathcal{M}_{0}=\operatorname{int}(\mathcal{K})$ and $\mathcal{M}_{1}=\partial \mathcal{K}$.

We recall that the value function of the Mayer problem with given final horizon $T>0$ is defined via

$$
\vartheta(t, x):=\inf \left\{\psi(y(T)) \mid \quad y \in \mathcal{S}_{t}^{T}(x)\right\}, \quad \forall(t, x) \in[0, T] \times \mathcal{K},
$$

where $\mathcal{S}_{a}^{b}(x)$ stands for the set of admissible trajectories solution of $(\mathrm{DI})_{\mathcal{K}}$ defined on the interval $[a, b]$ with initial condition $y(a)=x$.

In the sequel, we shall also use the notation $\mathcal{S}^{T}$ for the set of all admissible trajectories starting at any position in $\mathcal{K}$ and at any time in $[0, T]$ :

$$
\mathcal{S}^{T}:=\left\{y \in \mathcal{S}_{t}^{T}(x), \text { for } t \in[0, T], x \in \mathcal{K}\right\} .
$$

All along this paper we assume that the final cost $\psi: \mathbb{R}^{N} \rightarrow \mathbb{R} \cup\{+\infty\}$ satisfies the following:

$$
\psi(\cdot) \text { is l.s.c. and bounded from below on } \mathcal{K} \text {. }
$$

Remark 2.2 In the formulation of the Mayer problem it is possible to consider implicitly a final constraint $\Theta \subseteq \mathbb{R}^{N}$ of the form

$$
y(T) \in \Theta, \quad \forall y \in \mathcal{S}_{t}^{T}(x) .
$$

To do this, it is enough to replace $\psi$ with $\psi_{\Theta}: \mathbb{R}^{N} \rightarrow \mathbb{R} \cup\{+\infty\}$ defined by

$$
\psi_{\Theta}(x):=\left\{\begin{array}{ll}
\psi(x) & \text { if } x \in \Theta, \\
+\infty & \text { otherwise, }
\end{array} \quad \forall x \in \mathbb{R}^{N} .\right.
$$

If $\Theta$ is a closed set and $\left(H_{\psi}\right)$ holds, then $\psi_{\Theta}$ verifies $\left(H_{\psi}\right)$ as well.

The set of dynamics $F: \mathbb{R}^{N} \rightrightarrows \mathbb{R}^{N}$ is initially taken as to verify

$$
\left\{\begin{array}{l}
\text { i) } \quad F \text { is upper semicontinuous on } \mathbb{R}^{N} . \\
\text { ii) } F \text { has nonempty compact convex images on a neighborhood of } \mathcal{K} . \\
\text { iii) } \exists c_{F}>0 \text { so that } \max \{|v| \mid v \in F(x)\} \leq c_{F}(1+|x|), \forall x \in \mathcal{K} .
\end{array}\right.
$$

In the light of the standard theory of differential inclusions, the assumptions over the dynamics guarantee, for every $(t, x) \in[0, T) \times \mathcal{K}$, the existence of $\delta>0$ and an absolutely continuous curve $y:[t, t+\delta] \rightarrow \mathbb{R}^{N}$ which solves

$$
\dot{y}(s) \in F(y(s)), \quad \text { for a.e. } s \in[t, t+\delta], \quad y(t)=x .
$$

The foregoing trajectory may not be feasible, not even for small times. Anyhow, if the trajectory lives in $\mathcal{K}$ on the interval $[t, t+\delta]$, then the Gronwall's Lemma leads to

$$
|y(s)| \leq(1+|x|) e^{c_{F}(t-s)}, \quad \forall s \in[t, t+\delta] .
$$




\subsection{The value function and compatibility assumptions}

It is well-known that the Value Function is likely to be l.s.c. as long as the dynamics maps has convex images. The next proposition provides a precise statement for the present setting.

Proposition 2.1 Suppose $\mathcal{K}$ is a closed subset of $\mathbb{R}^{N}$ and that $\left(H_{\psi}\right)$ as well as $\left(H_{F}\right)$ is satisfied. Then, if $\vartheta(t, x) \in \mathbb{R}$ there exists an optimal trajectory $\bar{y} \in \mathcal{S}_{t}^{T}(x)$ for the Mayer problem. Furthermore, $\vartheta:[0, T] \times \mathcal{K} \rightarrow \mathbb{R} \cup\{+\infty\}$ is l.s.c. and is bounded from below.

This proposition is a classical result and is it basically due to the compactness of trajectories of the system. For this reason we only provide a sketch of the proof.

Proof. [Sketch of the proof of Proposition 2.1] Let $(t, x) \in[0, T] \times \mathcal{K}$ so that $\vartheta(t, x) \in \mathbb{R}$. The estimate (2.1) together with [5, Theorem 0.3.4] and the Convergence Theorem (cf. [5, Theorem 1.4.1]) yield to the existence of a minimizing subsequence that converges uniformly to some $\bar{y} \in \mathcal{S}_{t}^{T}(x)$ and whose weak derivative converges weakly to $\dot{\bar{y}}$ in $L^{1}\left([t, T], \mathbb{R}^{N}\right)$. Thus, since $\psi$ is l.s.c. the optimality of $\bar{y}$ follows easily.

For the lower semicontinuity of the Value Function, if $\left\{\left(t_{n}, x_{n}\right)\right\} \subseteq$ dom $\vartheta$ converges to some $(t, x)$, it is enough to take $y_{n} \in \mathcal{S}_{t_{n}}^{T}\left(x_{n}\right)$ optimal and use the same compactness arguments as above to prove that $y_{n}$ has a subsequence that converges to an element of $\mathcal{S}_{t}^{T}(x)$ and then conclude the proof using the definition of the $\vartheta$.

Before going further, we require to introduce some notation and to disclose the compatibility assumptions under which the main theorem is stated. Let $\mathcal{T}_{\mathcal{K}}^{B}(\cdot)$ stands for the Bouligand tangent cone to $\mathcal{K}$ which is given by

$$
\mathcal{T}_{\mathcal{K}}^{B}(x)=\left\{v \in \mathbb{R}^{N} \mid \liminf _{t \rightarrow 0^{+}} \frac{\operatorname{dist}_{\mathcal{K}}(x+t v)}{t}=0\right\}, \quad \forall x \in \mathcal{K} .
$$

In the main result of the paper we are going to relate the supersolution principle with a smaller Hamiltonian than the classical one. For this purpose, we write $F^{\sharp}: \mathcal{K} \rightrightarrows \mathbb{R}^{N}$ for the multivalued map defined via

$$
F^{\sharp}(x):=F(x) \cap \mathcal{T}_{\mathcal{K}}^{B}(x), \quad \forall x \in \mathcal{K} .
$$

On the other hand, the subsolution principle will be associated with a different Hamiltonian on each stratum. Let us define, for each index $i \in \mathcal{I}$, the multifunction $F_{i}: \mathcal{M}_{i} \rightrightarrows \mathbb{R}^{N}$ as follows

$$
F_{i}(x):=F(x) \cap \mathcal{T}_{\mathcal{M}_{i}}(x), \quad \forall x \in \mathcal{M}_{i} .
$$

In accordance with the definitions introduced in [20] for parametrized vector fields, we call this setvalued map the tangential dynamics to $\mathcal{M}_{i}$.

Remark 2.3 Note that $\mathcal{T}_{\mathcal{M}_{i}}(\cdot)$ has always closed graph on each closed subset of $\mathcal{M}_{i}$. Furthermore, since $F(\cdot)$ is upper semicontinuous all along $\mathcal{K}$, the multifunction $F_{i}(\cdot)$ turns out to be upper semicontinuous on $\mathcal{M}_{i}$. Furthermore, its images are compact convex, potentially nonempty, sets of $\mathbb{R}^{N}$.

At present, the tangential dynamics to a stratum play a similar role as the tangents controls in introduced in [20]. Consequently, all the theory we develop from this point on is done under the following assumption:

Each $F_{i}$ is locally Lipschitz continuous on $\mathcal{M}_{i}$ w.r.t. the Hausdorff distance.

We recall that we have adopted the convention $d_{H}(\emptyset, S)=+\infty$ for $S \neq \emptyset$. Thus, $\left(H_{1}\right)$ implies that the images of $F_{i}(\cdot)$ are either empty or nonempty throughout $\mathcal{M}_{i}$. 
Remark 2.4 We would like to stress that the domain $\mathcal{K}$ may not be given a priori such a stratified partition, but we assume with $\left(H_{0}\right)$ and $\left(H_{1}\right)$ that there is at least one such partition.

Moreover, for technical reasons we also require a controllability condition on certain strata. We evoke that the reachable set of the control system, which is written as $\mathcal{R}(t, x ; s)$, is the set of points that can be attained at time $s$ with an admissible trajectory solution of (DI) $)_{\mathcal{K}}$ having initial condition $y(t)=x$. We consider in addition $\mathcal{R}_{i}(t, x ; \cdot)$ as the reachable set through the stratum $\mathcal{M}_{i}$, that is, the set of all possible positions that can be reached with an admissible arc lying entirely in $\mathcal{M}_{i}$ :

$$
\mathcal{R}_{i}(t, x ; s):=\bigcup_{y \in \mathcal{S}_{t}^{s}(x)}\left\{y(s) \mid y(\tau) \in \mathcal{M}_{i}, \forall \tau \in[t, s)\right\}, \quad \forall x \in \mathcal{M}_{i}, \forall t, s \in \mathbb{R}, t<s .
$$

Therefore, the controllability hypothesis that will be required in this paper is stated as follows:

$$
\left\{\begin{array}{l}
\forall \rho>0, \forall i \in \mathcal{I}, \text { if dom } F_{i} \neq \emptyset, \text { then } \exists \varepsilon_{i}, \Delta_{i}>0 \text { so that } \forall x \in \mathcal{M}_{i} \cap \mathbb{B}_{\rho} \\
\mathcal{R}(t, x ; s) \cap \overline{\mathcal{M}}_{i} \subseteq \bigcup_{r \in\left[t, t+\Delta_{i} s\right]} \mathcal{R}_{i}(t, x ; r), \forall t \in[0, T], \forall s \in\left[t, t+\varepsilon_{i}\right] .
\end{array}\right.
$$

This assumption is made in order to approximate curves that may switch between two or more strata infinitely many times on a short interval (see Lemma 3.3 below).

Remark 2.5 Note that $\left(\mathrm{H}_{2}\right)$ is trivial if $\mathcal{M}_{i}$ is an open set or more generally if $\mathcal{M}_{i}$ is of maximal dimension among the strata of $\mathcal{K}$ (indeed, in this case there exists $\varepsilon>0$ such that for any $\tau \in[0, \varepsilon]$, we have: $\left.\mathcal{R}(t, x ; t+\tau) \cap \overline{\mathcal{M}}_{i}=\mathcal{R}_{i}(t, x ; t+\tau)\right)$. The same remark holds whenever $F_{i}=F$. Moreover, $\left(H_{2}\right)$ is straightforward if $\mathcal{M}_{i}$ is a single point (since in this case, if $F_{i} \neq \emptyset$ then $F_{i} \equiv\{0\}$ and $\left.\mathcal{R}(t, x ; s) \cap \overline{\mathcal{M}}_{i}=\overline{\mathcal{M}}_{i}=\mathcal{R}_{i}(t, x ; s)\right)$.

Assumption $\left(H_{2}\right)$ may seem rigid at first sight but it is somehow the minimum requirement needed if the value function is discontinuous. There are some easy-to-verify criteria that can be used to check if assumption $\left(H_{2}\right)$ holds. For example, a projection condition over strata $\mathcal{M}_{i}$, for every $i \in \mathcal{I}$ such that $\operatorname{dom} F_{i} \neq \emptyset$ :

$$
\forall \bar{x} \in \mathcal{M}_{i}, \exists r>0 \text { such that } D \Pi_{i}(x) \cdot v \in F(x), \quad \forall x \in \mathbb{B}(\bar{x}, r) \cap \mathcal{K}, \forall v \in F(x),
$$

where $\Pi_{i}(x)$ stands for the projection of $x$ over the manifold $\mathcal{M}_{i}$ and $D \Pi_{i}(x)$ stands for the derivative of $\Pi_{i}$ on $x$. We recall that $x \mapsto \Pi_{i}(x)$ is well defined and differentiable on a tubular neighborhood around $\mathcal{M}_{i}$; see for instance [22, Theorem 6.24]. Furthermore, it follows by definition that for any $x$ in the tubular neighborhood we have

$$
D \Pi_{i}(x): \mathbb{R}^{n} \rightarrow \mathcal{T}_{\mathcal{M}_{i}}(x)
$$

and so if $D \Pi_{i}(x) \cdot v \in F(x)$, then we actually have that $D \Pi_{i}(x) \cdot v \in F_{i}(x)$. Therefore, if the projection condition (2.2) is satisfied, then for $y \in \mathcal{S}_{t}^{s}(x)$ with $y(s) \in \mathcal{M}_{i}$, setting $\tilde{y}(\tau):=\Pi_{i}(y(\tau))$ for $\tau \in[t, s]$, it comes that $\tilde{y} \in \mathcal{S}_{t}^{s}(x)$ with $y(s)=\tilde{y}(s)$, and $\tilde{y}(\tau) \in \mathcal{M}_{i}$ for any $\tau \in[t, s]$. In this case, $\left(H_{2}\right)$ holds with $\Delta_{i}=1$.

Let us also point out the fact that $\left(H_{2}\right)$ can be satisfied under an easy criterion of full controllability condition on manifolds. The most classical assumption of this kind of controllability is the following:

$$
\forall i \in \mathcal{I} \text { with dom } F_{i} \neq \emptyset . \exists r_{i}>0 \text { such that } \mathcal{T}_{\mathcal{M}_{i}}(x) \cap \mathbb{B}\left(0, r_{i}\right) \subseteq F_{i}(x), \quad \forall x \in \mathcal{M}_{i} .
$$

This criterion is a sufficient condition for $\left(H_{2}\right)$ to be fulfilled. Indeed, this corresponds to the a controllability condition on manifolds. Hence, by adapting the classical arguments to this setting, we can see that (2.3) implies the Lipschitz regularity of the minimum time function of the controlled dynamics restricted to the manifold $\mathcal{M}_{i}$, and so $\left(H_{2}\right)$ follows; see for instance [6, Chapter 4.1]. However, let us 
emphasize that preceding criterion is only a sufficient condition to satisfy assumption $\left(H_{2}\right)$. Moreover, $\left(H_{2}\right)$ has to be satisfied only on strata where a chattering occurs.

The characterization of the value function on this paper have to be understood in the bilateral sense. Accordingly, to do so, we recall the notion of viscosity subgradient.

Definition 2.1 Let $\omega: \mathbb{R}^{N} \rightarrow \mathbb{R} \cup\{+\infty\}$ be a given l.s.c. function. A vector $\zeta \in \mathbb{R}^{N}$ is called a viscosity subgradient of $\omega$ at $x \in$ dom $\omega$ if and only if there exists a continuously differentiable function $g: \mathbb{R}^{N} \rightarrow \mathbb{R}$ so that

$$
\nabla g(x)=\zeta \text { and } \omega-g \text { attains a local minimum at } x .
$$

The set of all viscosity subgradients at $x$ is denoted by $\partial_{V} \omega(x)$.

With this definition at hand and under the aforesaid assumptions, we can give a characterization of the value function.

Theorem 2.2 Suppose $\left(H_{0}\right),\left(H_{1}\right)$ and $\left(H_{2}\right)$ hold along with $\left(H_{\psi}\right)$ and $\left(H_{F}\right)$. Then the value function of the Mayer problem $\vartheta$ is the unique l.s.c. function on $\mathcal{K}$ which is $+\infty$ outside $\mathcal{K}$, bounded from below and that verifies

$$
\begin{gathered}
-\theta+\max _{v \in F^{\sharp}(x)}\{-\langle v, \zeta\rangle\} \geq 0, \quad \forall(t, x) \in[0, T) \times \mathcal{K}, \forall(\theta, \zeta) \in \partial_{V} \vartheta(t, x), \\
-\theta+\max _{v \in F_{i}(x)}\{-\langle v, \zeta\rangle\} \leq 0, \quad \forall i \in \mathcal{I}, \forall(t, x) \in(0, T] \times \mathcal{M}_{i}, \forall(\theta, \zeta) \in \partial_{V} \vartheta_{i}(t, x), \\
\vartheta(T, x)=\psi(x), \quad x \in \mathcal{K},
\end{gathered}
$$

where $\vartheta_{i}$ stands for the function that agrees with $\vartheta$ on $\overline{\mathcal{M}}_{i}$ and is $+\infty$ elsewhere.

The proof of Theorem 2.2 is composed of a part that is rather standard and another which uses stratified techniques. In any case, we make use of monotone properties of the value function along trajectories which for this case read as follows.

Definition 2.2 A function $\omega:[0, T] \times \mathcal{K} \rightarrow \mathbb{R} \cup\{+\infty\}$ is said to be:

i) weakly decreasing along trajectories of $\mathcal{S}^{T}$ provided for all $(t, x) \in$ dom $\omega$ we can find a curve $y \in \mathcal{S}_{t}^{T}(x)$ so that $\omega(s, y(s)) \leq \omega(t, x)$ for all $t \leq s \leq T$.

ii) strongly increasing along trajectories of $\mathcal{S}^{T}$ if for each $(t, x) \in[0, T] \times \mathcal{K}$ and each $y \in \mathcal{S}_{t}^{T}(x)$, we have $\omega(s, y(s)) \geq \omega(t, x)$ for all $t \leq s \leq T$.

The interest in the foregoing definition lies in the following comparison lemma.

Lemma 2.3 Let $\omega:[0, T] \times \mathcal{K} \rightarrow \mathbb{R} \cup\{+\infty\}$ be a given function satisfying $\omega(T, x)=\psi(x)$ for all $x \in \mathcal{K}$.

1. If $\omega$ is weakly decreasing along trajectories of $\mathcal{S}^{T}$, then $\vartheta(t, x) \leq \omega(t, x)$ for each $(t, x) \in[0, T] \times \mathcal{K}$.

2. If $\omega$ is strongly increasing along trajectories of $\mathcal{S}^{T}$, then $\vartheta(t, x) \geq \omega(t, x)$ for every $(t, x) \in$ $[0, T] \times \mathcal{K}$.

Proof. It is enough to evaluate each inequality at $s=T$, use the end-point condition and the definition of the value function $\vartheta$.

We evoke that the value function of the Mayer problem solves the functional equation usually referred as the Dynamic Programming Principle:

$$
\vartheta(t, x)=\inf \left\{\vartheta(s, y(s)) \mid \quad y \in \mathcal{S}_{t}^{T}(x)\right\}, \quad \forall x \in \mathcal{K}, \quad \forall 0 \leq t \leq s \leq T .
$$

The preceding lemma leads to assert, that the Value Function is the unique function being weakly decreasing and strongly increasing along trajectories of $\mathcal{S}^{T}$ at the same time. So, to prove Theorem 2.2 it suffices to find equivalent formulation for the monotone properties in terms of HJB inequalities. 


\subsection{An Example}

The main feature of the theory we propose in this paper is that the set of state-constraints admits a stratified structure, meaning that it can be decomposed into a locally finite family of embedded manifolds of $\mathbb{R}^{N}$. This framework allows us to treat broader classes of state-constraints than the neighboring feasible trajectory (NFT) approach because we do not need to impose the condition $\overline{\operatorname{int}(\mathcal{K})}=\mathcal{K}$ which is essential for the NFT theorems, see [19].

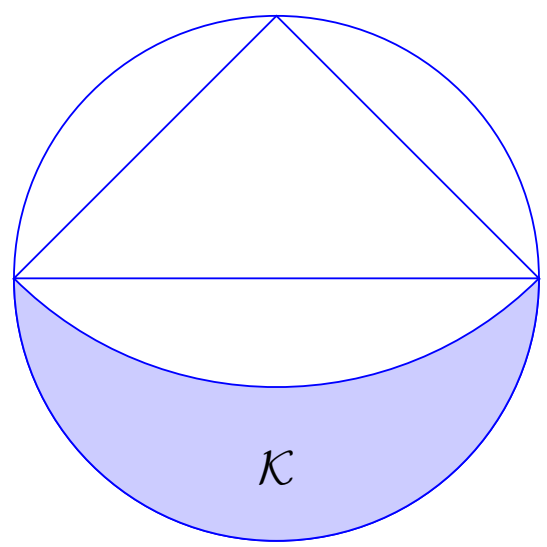

(a) A state-constraints set.

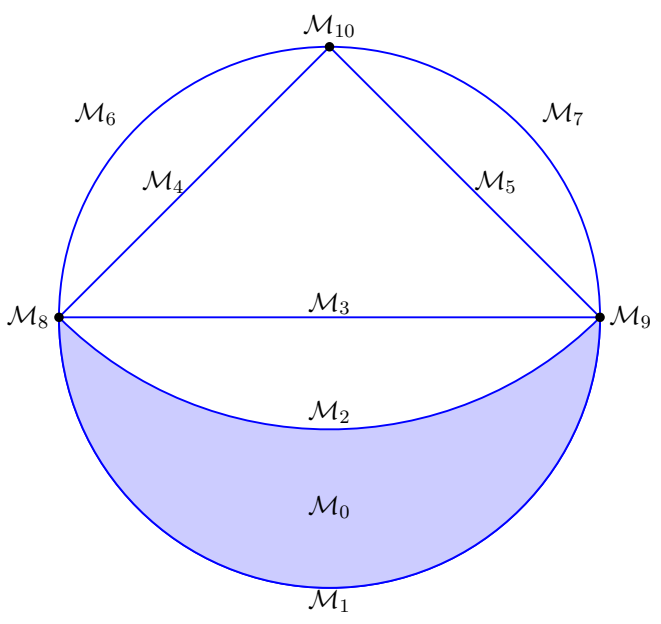

(b) A particular stratification.

Figure 1: An example of stratifiable state-constraints sets.

Figure 1a shows an example of state-constraints covered by our work, with a possible stratification illustrated in Figure $1 \mathrm{~b}$. Here $\mathcal{M}_{0}=\operatorname{int}(\mathcal{K}), \mathcal{M}_{1}, \ldots, \mathcal{M}_{7}$ are bounded curves and $\mathcal{M}_{8}, \mathcal{M}_{9}$ and $\mathcal{M}_{10}$ are single points. If $\left(H_{\psi}\right),\left(H_{1}\right)$ and $\left(H_{2}\right)$ hold for this stratification then, the system of HJB (2.4) implies that in particular the following inequalities hold on $\mathcal{M}_{0}$ :

$$
\left\{\begin{aligned}
-\theta+\max _{v \in F(x)}\{-\langle v, \zeta\rangle\} \geq 0, \quad \forall(t, x) \in[0, T) \times \mathcal{M}_{0}, \forall(\theta, \zeta) \in \partial_{V} \vartheta(t, x), \\
-\theta+\max _{v \in F(x)}\{-\langle v, \zeta\rangle\} \leq 0, \quad, \quad \forall(t, x) \in(0, T] \times \mathcal{M}_{0}, \quad \forall(\theta, \zeta) \in \partial_{V} \vartheta(t, x) .
\end{aligned}\right.
$$

With the same stratification considered in Figure 1b, the system (2.4) gives for $i=1,2$ :

$$
\begin{aligned}
& -\theta+\max _{v \in F^{\sharp}(x)}\{-\langle v, \zeta\rangle\} \geq 0, \forall(t, x) \in[0, T) \times \mathcal{M}_{i}, \forall(\theta, \zeta) \in \partial_{V} \vartheta(t, x), \\
& -\theta+\max _{v \in F_{i}(x)}\{-\langle v, \zeta\rangle\} \leq 0, \forall(t, x) \in(0, T] \times \mathcal{M}_{i}, \forall(\theta, \zeta) \in \partial_{V} \vartheta_{i}(t, x) .
\end{aligned}
$$

For the strata $i=3, \ldots, 7$, since $F^{\sharp}(x)=F_{i}(x)$ whenever $x \in \mathcal{M}_{i}$ and $\vartheta_{i}=\vartheta$ locally on $\mathcal{M}_{i}$, the system (2.4) yields to:

$$
\begin{aligned}
& -\theta+\max _{v \in F_{i}(x)}\{-\langle v, \zeta\rangle\}=0, \forall(t, x) \in(0, T) \times \mathcal{M}_{i}, \forall(\theta, \zeta) \in \partial_{V} \vartheta(t, x) . \\
& -\theta+\max _{v \in F_{i}(x)}\{-\langle v, \zeta\rangle\} \geq 0, \forall x \in \mathcal{M}_{i}, \forall(\theta, \zeta) \in \partial_{V} \vartheta(0, x) \\
& -\theta+\max _{v \in F_{i}(x)}\{-\langle v, \zeta\rangle\} \leq 0, \forall x \in \mathcal{M}_{i}, \forall(\theta, \zeta) \in \partial_{V} \vartheta(T, x)
\end{aligned}
$$

Finally, for $i=8,9,10$ when $F_{i}(x) \neq \emptyset$ then $F_{i}(x)=\{0\}$ on $\mathcal{M}_{i}$, and we have

$$
\begin{aligned}
-\theta+\max _{v \in F^{\sharp}(x)}\{-\langle v, \zeta\rangle\} & \geq 0, \quad \forall(t, x) \in[0, T) \times \mathcal{M}_{i}, \forall(\theta, \zeta) \in \partial_{V} \vartheta(t, x), \\
\theta & \geq 0, \quad \forall(t, x) \in(0, T] \times \mathcal{M}_{i}, \forall(\theta, \zeta) \in \partial_{V} \vartheta_{i}(t, x) .
\end{aligned}
$$




\section{Characterization of the monotone principles}

We begin this section with the most standard part of the proof of Theorem 2.2, namely, the supersolution principle. After doing so, we address our attention to the subsolution principle which is slightly more technical than the supersolution principle.

\subsection{Decreasing principle}

We now address the characterization of the weakly decreasing property in terms of a HJB inequality. We evoke once again that this part is rather classical, in particular no stratification of the stateconstraints interferes. However, the novelty on the statement is that the equation is written with a smaller Hamiltonian that only consider the viable velocities, i.e., those that belong to the Bouligand tangent cone to $\mathcal{K}$.

We begin with presenting (without proving) a result which is customary and well-known, that is, the characterization of the weakly decreasing principle by means of the usual Hamiltonian. The next lemma can be proved using the same scheme as for [20, Proposition 5.1]. For the unconstrained framework its proof can be found in $[17,18,13,28]$.

Lemma 3.1 Suppose $\mathcal{K}$ is closed and $\left(H_{\psi}\right)$ holds along with $\left(H_{F}\right)$. Consider a l.s.c. function $\omega$ : $[0, T] \times \mathcal{K} \rightarrow \mathbb{R} \cup\{+\infty\}$ verifying $\omega(T, x)=\psi(x)$ for all $x \in \mathcal{K}$. Then $\omega$ is weakly decreasing along trajectories of $\mathcal{S}^{T}$ if and only if

$$
-\theta+\mathcal{H}(x, \zeta) \geq 0 \quad \text { for all }(\theta, \zeta) \in \partial_{V} \omega(t, x), \quad \forall(t, x) \in[0, T) \times \mathcal{K},
$$

where the Hamiltonian is defined by $\mathcal{H}(x, \zeta):=\sup _{v \in F(x)}-\langle v, \zeta\rangle$.

We now show that a function verifies the weakly decreasing property if and only it is a supersolution of the HJB equation associated with the dynamics $F^{\sharp}$.

Proposition 3.2 Suppose $\mathcal{K}$ is closed and $\left(H_{\psi}\right)$ holds along with $\left(H_{F}\right)$. Consider a l.s.c. function $\omega:[0, T] \times \mathcal{K} \rightarrow \mathbb{R} \cup\{+\infty\}$ verifying $\omega(T, x)=\psi(x)$ for all $x \in \mathcal{K}$. Then $\omega$ is weakly decreasing along trajectories of $\mathcal{S}^{T}$ if and only if

$$
-\theta+\max _{v \in F^{\sharp}(x)}\{-\langle v, \zeta\rangle\} \geq 0 \quad \text { for all }(\theta, \zeta) \in \partial_{V} \omega(t, x), \quad \forall(t, x) \in[0, T) \times \mathcal{K} .
$$

Proof. Notice first that if (3.2) holds then (3.1) is satisfied too, because of $F^{\sharp}(x) \subseteq F(x)$ for any $x \in \mathcal{K}$. Consequently, the sufficient implication holds immediately by means of Lemma 3.1.

Hence, it only remains to show that $\omega$ being weakly decreasing along trajectories of $\mathcal{S}^{T}$ implies that (3.2) holds. If $\omega(t, x)=+\infty$, then $\partial_{V} \omega(t, x)=\emptyset$ meaning that (3.2) is trivial. So we might exclusively assume $\omega(t, x)<\infty$.

Let $(\theta, \zeta) \in \partial_{V} \omega(t, x)$, then in particular, by [13, Proposition 4.12] we have that for any sequence $\left\{\left(s_{n}, x_{n}\right)\right\}$ converging to $(t, x)$ the following holds true:

$$
\liminf _{n \rightarrow+\infty} \frac{\omega\left(s_{n}, x_{n}\right)-\omega(t, x)-\theta\left(s_{n}-t\right)-\left\langle\zeta, x_{n}-x\right\rangle}{\left|x_{n}-x\right|+\left|s_{n}-t\right|} \geq 0 .
$$

By the weak decreasing property, there is $y \in \mathcal{S}_{t}^{T}(x)$ so that

$$
\omega(s, y(s)) \leq \omega(t, x) \text { for all } t \leq s \leq T .
$$

Now choose any sequence $\left\{s_{n}\right\} \subseteq(t, T]$ so that $s_{n} \rightarrow t$ and $v_{n}:=\frac{y\left(s_{n}\right)-x}{s_{n}-t} \rightarrow v$. It is clear that $y\left(s_{n}\right) \rightarrow x$. This is always possible because $F$ is locally bounded.

We claim that $v \in F^{\sharp}(x)$. To see this notice that

$$
\int_{0}^{1} \gamma_{n}(\lambda) d \lambda=v_{n} \rightarrow v, \quad \text { with } \gamma_{n}(\lambda):=\dot{y}\left(\lambda s_{n}+(1-\lambda) t\right)
$$


Take $\varepsilon>0$ arbitrary. Since $F$ is upper semicontinuous at $x$ there is $n_{\varepsilon} \in \mathbb{N}$ so that

$$
F\left(y\left(\lambda s_{n}+(1-\lambda) t\right)\right) \subseteq F(x)+\overline{\mathbb{B}}(0, \varepsilon), \quad \forall n \geq n_{\varepsilon}, \forall \lambda \in[0,1]
$$

Since $F(x)+\overline{\mathbb{B}}(0, \varepsilon)$ is a compact convex set and $\gamma_{n}(\lambda) \in F\left(y\left(\lambda s_{n}+(1-\lambda) t\right)\right)$ a.e. on [0, 1$]$, by [27, Lemma 4.2] we have that

$$
v_{n}=\int_{0}^{1} \gamma_{n}(\lambda) d \lambda \in F(x)+\overline{\mathbb{B}}(0, \varepsilon), \quad \forall n \geq n_{\varepsilon} .
$$

Letting $n \rightarrow+\infty$ we find out that $v \in F(x)+\overline{\mathbb{B}}(0, \varepsilon)$. Moreover, since $\varepsilon>0$ is arbitrary, we get that $v \in \overline{F(x)}=F(x)$.

Furthermore, since $y\left(s_{n}\right) \in \mathcal{K}$ for all $n \in \mathbb{N}$, one has $v \in F(x) \cap \mathcal{T}_{\mathcal{K}}^{B}(x)=F^{\sharp}(x)$, so the claim holds true.

Now, setting $x_{n}=y\left(s_{n}\right)$ and using (3.4) we get

$$
\frac{\omega\left(s_{n}, x_{n}\right)-\omega(t, x)-\theta\left(s_{n}-t\right)-\left\langle\zeta, x_{n}-x\right\rangle}{\left|x_{n}-x\right|+\left|s_{n}-t\right|} \leq \frac{-\theta\left(s_{n}-t\right)-\left\langle\zeta, x_{n}-x\right\rangle}{\left|x_{n}-x\right|+\left|s_{n}-t\right|}
$$

Besides, it is not difficult to see that

$$
\frac{-\theta\left(s_{n}-t\right)-\left\langle\zeta, x_{n}-x\right\rangle}{\left|x_{n}-x\right|+\left|s_{n}-t\right|} \rightarrow \frac{-\theta-\langle\zeta, v\rangle}{|v|+1}
$$

Thus, by virtue of (3.3), letting $n \rightarrow \infty$ in (3.5), we find out that

$$
-\theta+\max _{v \in F^{\sharp}(x)}\{-\langle v, \zeta\rangle\} \geq-\theta-\langle v, \zeta\rangle \geq 0 .
$$

Thus, given that $(t, x)$ and $(\theta, \zeta)$ are arbitrary the conclusion follows.

\subsection{Increasing principle}

The last step required in the proof of Theorem 2.2 is the characterization of strongly increasing functions along the trajectories of the controlled system. The following result is the corresponding version for the Mayer problem of [20, Proposition 5.2 and Proposition 5.3].

We would like to stress on that the necessary condition in the next proposition holds under weaker assumptions. Actually, the controllability assumption is not al all required (as in [20, Proposition 5.2]) and the Lipschitz continuity hypothesis $\left(H_{1}\right)$ can be relaxed to lower semicontinuity with nonempty images.

On the other hand, for the sufficient implication we do require the controllability assumption. As a matter of fact, the following lemma, whose proof strongly relies on $\left(H_{2}\right)$ is fundamental for the proof of Proposition 3.5 below.

Lemma 3.3 Suppose $\left(H_{0}\right),\left(H_{1}\right)$ and $\left(H_{2}\right)$ hold. Let $(t, x) \in[0, T] \times \mathcal{K}$ and $y \in \mathcal{S}_{t}^{T}(x)$ be given, then for any $\varepsilon>0$ and $\tau \in[t, T]$ we can find $x_{\varepsilon} \in \mathbb{B}(x, \varepsilon) \cap \mathcal{K}, t_{\varepsilon} \in(t-\varepsilon, t+\varepsilon) \cap[0, \tau]$ and $y_{\varepsilon} \in \mathcal{S}_{t_{\varepsilon}}^{\tau}\left(x_{\varepsilon}\right)$ that verifies $y_{\varepsilon}(\tau)=y(\tau)$ and that is regular in the following sense:

There exists a partition of $[t, \tau],\left\{t=t_{0}<t_{1}<\ldots<t_{n}<t_{n+1}=\tau\right\}$, so that for any $l \in\{0, \ldots, n\}$ we can find $i \in \mathcal{I}$ such that $y_{\varepsilon}(s) \in \mathcal{M}_{i}$ on $\left(t_{l}, t_{l+1}\right)$

The proof of the above-stated lemma is perhaps the most technical reasoning in the paper. For sake of the exposition we have left its proof for the end and we now focus on the characterization of the suboptimality principle. 


\subsubsection{Some background in nonsmooth analysis}

In the sequel, most of time we are going to work with a subfamily of viscosity subgradients called the proximal ones whose definition we recall below.

Definition 3.1 Let $\omega: \mathbb{R}^{N} \rightarrow \mathbb{R} \cup\{+\infty\}$ be a given l.s.c. function. A viscosity subgradient $\zeta \in \mathbb{R}^{N}$ of $\omega$ at $x \in$ dom $\omega$ is called a proximal subgradient of $\omega$ at $x$ if for some $\sigma>0$ the test function $g: \mathbb{R}^{N} \rightarrow \mathbb{R}$ can be taken as

$$
g(y):=\langle\zeta, y-x\rangle-\sigma|y-x|^{2}, \quad \forall y \in \mathbb{R}^{N} .
$$

The set of all proximal subgradients at $x$ is denoted by $\partial_{P} \omega(x)$.

For sake of the exposition, we evoke the definition of the Proximal normal cone and its relation with the proximal subgradients. For a further discussion about this topic we refer the reader to [13].

Let $\mathcal{E} \subseteq \mathbb{R}^{k}$ be a locally closed set and let $x \in \mathcal{E}$. A vector $\eta \in \mathbb{R}^{k}$ is called proximal normal to $\mathcal{E}$ at $x$ if there exists $\sigma=\sigma(x, \eta)>0$ so that

$$
\frac{|\eta|}{2 \sigma}|x-y|^{2} \geq\langle\eta, y-x\rangle \quad \forall y \in \mathcal{E}
$$

The set of all such vectors $\eta$ is known as the Proximal normal cone to $\mathcal{E}$ at $x$ and is denoted by $\mathcal{N}_{\mathcal{E}}^{P}(x)$. If $\mathcal{E}=$ epi $\omega$ where $\omega: \mathbb{R}^{k} \rightarrow \mathbb{R} \cup\{+\infty\}$ is a l.s.c. function, then for every $x \in \operatorname{dom} \omega$, the following relation is valid:

$$
\xi \in \partial_{P} \omega(x) \quad \Leftrightarrow \quad(\xi,-1) \subseteq \mathcal{N}_{\mathcal{E}}^{P}(x, \omega(x)), \quad \forall x \in \operatorname{dom} \omega .
$$

We now present a useful criterion for strong invariance adapted to smooth manifolds whose proof can be found in [20].

Lemma 3.4 ([20, Proposition 4.2]) Suppose $M \subseteq \mathbb{R}^{k}$ is locally closed, $\mathcal{E} \subseteq \mathbb{R}^{k}$ is closed with $\mathcal{E} \cap \bar{M} \neq \emptyset$ and $\Gamma: \bar{M} \rightrightarrows \mathbb{R}^{k}$ is locally Lipschitz and locally bounded.

Let $r>0$ and assume that there exists $\kappa=\kappa(r)>0$ such that

$$
\sup _{\nu \in \Gamma(x)}\langle x-s, \nu\rangle \leq \kappa \operatorname{dist}_{\mathcal{E} \cap \bar{M}}(x)^{2} \quad \forall x \in M \cap \mathbb{B}_{r}, \forall s \in \operatorname{proj}_{\mathcal{E} \cap \bar{M}}(x) .
$$

Then for any absolutely continuous arc $\gamma:[0, T] \rightarrow \bar{M}$ that satisfies

$$
\dot{\gamma} \in \Gamma(\gamma) \quad \text { a.e. on }[0, T] \quad \text { and } \quad \gamma(t) \in M \cap \mathbb{B}_{r} \quad \forall t \in(0, T),
$$

the following estimate holds true

$$
\operatorname{dist}_{\mathcal{E} \cap \bar{M}}(\gamma(t)) \leq e^{\kappa t} \operatorname{dist}_{\mathcal{E} \cap \bar{M}}(\gamma(0)) \quad \forall t \in[0, T] .
$$

\subsubsection{Characterization of the suboptimality principle}

We are now in position to provide and proof the the characterization of the suboptimality principle which reads as follows.

Proposition 3.5 Suppose $\left(H_{0}\right),\left(H_{1}\right)$ and $\left(H_{2}\right)$ hold along with $\left(H_{\psi}\right)$ and $\left(H_{F}\right)$. Consider a l.s.c. function $\omega:[0, T] \times \mathcal{K} \rightarrow \mathbb{R} \cup\{+\infty\}$ verifying $\omega(T, x)=\psi(x)$ for all $x \in \mathcal{K}$. Then $\omega$ is strongly increasing along trajectories of $\mathcal{S}^{T}$ if and only if

$$
-\theta+\max _{v \in F_{i}(x)}\{-\langle v, \zeta\rangle\} \leq 0 \quad \forall i \in \mathcal{I}, \forall(t, x) \in(0, T] \times \mathcal{K},(\theta, \zeta) \in \partial_{V} \omega_{i}(t, x),
$$

where $\omega_{i}=\omega$ over $\overline{\mathcal{M}}_{i}$ and $+\infty$ elsewhere. 
Proof. Notice first that if $\operatorname{dom} F_{i}=\emptyset$ for some $i \in \mathcal{I}$, then (3.8) does not provide any information and holds trivially.

The implication $(\Rightarrow)$ is proven first. Take $i \in \mathcal{I}$ so that $\operatorname{dom} F_{i} \neq \emptyset$, then $\left(H_{1}\right)$ implies that $F_{i}$ is locally Lipschitz continuous on $\mathcal{M}_{i}$. Consequently, it is l.s.c. and its images are nonempty compact convex sets of $\mathbb{R}^{N}$. Take $(t, x) \in(0, T] \times \mathcal{M}_{i}$ and $v \in F_{i}(x)$ fixed but arbitrary. By the Michael's Selection Theorem ([5, Theorem 1.11.1]), there exists a continuous selection $f_{i}$ of $F_{i}$ that verifies $f_{i}(x)=v$. By the Nagumo's Theorem ([5, Theorem 4.2.2]), there exist $\delta>0$ with $t-\delta \geq 0$ and a continuously differentiable trajectory of the control system $y \in \mathcal{S}_{t-\delta}^{t+\delta}(x)$ that verifies $\dot{y}(s)=f_{i}(y(s))$ and $y(s) \in \mathcal{M}_{i}$ for any $s \in(t-\delta, t+\delta)$.

Suppose $\partial_{V} \omega_{i}(t, x) \neq \emptyset$, otherwise, (3.8) is immediately satisfied. Under these circumstances, $\vartheta(t, x) \in \mathbb{R}$ and so $\mathcal{S}_{t}^{T}(x) \neq \emptyset$. Take $\bar{y} \in \mathcal{S}_{t}^{T}(x)$ and remark that $\tilde{y}=y \mathbb{1}_{[t-\delta, t)}+\bar{y} \mathbb{1}_{[t, T]} \in \mathcal{S}_{t-\delta}^{T}(y(t-\delta))$. Therefore, if $\omega$ is strongly increasing we have $\omega(t, x) \geq \omega(s, y(s))$ for any $s \in[t-\delta, t]$.

Let $(\theta, \zeta) \in \partial_{P} \omega_{i}(t, x)$, the proximal inequality combined with the monotone property yields to

$$
\theta+\left\langle\frac{x-y(s)}{t-s}, \zeta\right\rangle \geq \sigma\left[(s-t)+|y(s)-x|\left|\frac{x-y(s)}{t-s}\right|\right],
$$

for some $\sigma>0$ and for any $s \in(t-\delta, t)$ close to $t$; this is due to $y(s) \in \mathcal{M}_{i}$ for each $s \in[t-\delta, t]$. Notice also that

$$
\frac{x-y(s)}{t-s}=\int_{0}^{1} f_{i}\left(y\left(\lambda s_{n}+(1-\lambda) t\right)\right) d \lambda \rightarrow v, \quad \text { if } s \rightarrow t \text { with } s<t .
$$

Hence, letting $s \rightarrow t$ and noticing that $v \in F_{i}(x)$ is arbitrary we get (3.8) for any proximal subgradient. The extension to viscosity subgradients is a consequence of [13, Proposition 3.4.5]. Indeed, if $(\theta, \zeta) \in$ $\partial_{V} \omega(t, x) \backslash \partial_{P} \omega(t, x)$ there exist some sequence $\left\{\left(t_{n}, x_{n}\right)\right\} \subseteq[0, T] \times \mathcal{K}$ and $\left\{\left(\theta_{n}, \zeta_{n}\right)\right\} \subseteq \mathbb{R} \times \mathbb{R}^{N}$ so that

$$
\left(t_{n}, x_{n}, \theta_{n}, \zeta_{n}\right) \rightarrow(t, x, \theta, \zeta) \quad \text { and } \quad\left(\theta_{n}, \zeta_{n}\right) \in \partial_{P} \omega\left(t_{n}, x_{n}\right) .
$$

By the earlier reasoning, we have

$$
-\theta_{n}-\left\langle v_{n}, \zeta_{n}\right\rangle \leq 0 \quad \forall v_{n} \in F_{i}\left(x_{n}\right), \forall n \in \mathbb{N} .
$$

Moreover, since $F_{i}$ is lower semicontinuous on $\mathcal{M}_{i}$, for any $v \in F_{i}(x)$ there exists a sequence $v_{n} \rightarrow v$ with $v_{n} \in F_{i}\left(x_{n}\right)$. Evaluating the last inequality at this sequence $\left\{v_{n}\right\}$ and letting $n \rightarrow+\infty$ we obtain (3.8) for any viscosity subgradient, and so, the necessary implication holds.

We divide the rest of the proof for the sufficiency of (3.8) in several claims.

Claim A: If $i \in \mathcal{I}$ with dom $F_{i} \neq \emptyset$, then for each $(t, x) \in \operatorname{dom} \vartheta, \tau \in(t, T]$ and $y \in \mathcal{S}_{t}^{\tau}(x)$ for which $y(s) \in \mathcal{M}_{i}$ for all $s \in(t, \tau)$, we have $\omega(\tau, y(\tau)) \geq \omega(t, x)$.

Proof. [Claim A] Set $\mathcal{E}_{i}=$ epi $\left(\omega_{i}\right)$ and $M_{i}=\mathbb{R} \times \mathcal{M}_{i} \times \mathbb{R}$ and consider the multifunction $\Gamma_{i}(t, x, z)=$ $\{-1\} \times-F_{i}(x) \times\{0\}$ for any $(t, x, z) \in[0, T] \times \mathcal{M}_{i} \times \mathbb{R}$.

Note that $M_{i}$ is an embedded manifold of $\mathbb{R}^{N+2}$ and $\Gamma_{i}$ satisfies the same assumptions as $F_{i}$. Then if (3.8) holds, we claim that the following also holds

$$
\sup _{\nu \in \Gamma_{i}(t, x, z)}\langle\eta, \nu\rangle \leq 0 \quad \forall(t, x, z) \in \mathcal{E}_{i}, \forall \eta \in \mathcal{N}_{\mathcal{E}_{i}}^{P}(t, x, z) .
$$

Indeed, if $\mathcal{E}_{i}=\emptyset$ it holds by vacuity. Otherwise, take $(t, x, z) \in \mathcal{E}_{i}$ and a proximal normal $(\xi,-p) \in$ $\mathcal{N}_{\mathcal{E}_{i}}^{P}(t, x, z)$. Therefore, we have $p \geq 0$ because $\mathcal{E}_{i}$ is the epigraph of a function. Recall that $\Gamma_{i}(t, x, z) \neq$ 
$\emptyset$ because $F_{i}(x) \neq \emptyset$. Consider $p>0$, then $z=\omega_{i}(t, x)$ and by $(3.6)$, there is $(\theta, \zeta) \in \partial_{P} \omega_{i}(t, x)$ such that $\xi=(p \theta, p \zeta)$. Hence, for any $\nu \in \Gamma_{i}(t, x, z)$ we have, for some $v \in F_{i}(x)$, the following

$$
\langle(\xi,-p), \nu\rangle=p(-\theta-\langle\zeta, v\rangle) \leq p\left(-\theta+\sup _{v \in F_{i}(x)}\{-\langle\zeta, v\rangle\}\right) .
$$

Since (3.8) holds and $\nu \in \Gamma_{i}(t, x, z)$ is arbitrary, we can take supremum over $v$ to obtain the desired inequality (3.10). If on the other hand $p=0$ by Rockafellar's Theorem (cf. [12, Theorem 11.30]) there exist some sequences $\left\{\left(t_{n}, x_{n}\right)\right\} \subseteq \operatorname{dom} \omega_{i},\left\{\left(\xi_{n}\right)\right\} \subseteq \mathbb{R}^{N+1}$ and $\left\{p_{n}\right\} \subseteq(0, \infty)$ such that

$$
\left(t, x_{n}, \xi_{n}, p_{n}\right) \rightarrow(t, x, \xi, 0), \quad \omega_{i}\left(t_{n}, x_{n}\right) \rightarrow \omega(t, x), \quad \frac{1}{p_{n}} \xi_{n} \in \partial_{P} \omega_{i}\left(t_{n}, x_{n}\right) .
$$

Thus, using the same argument as above we can show

$$
\sup _{\nu \in \Gamma_{i}\left(t_{n}, x_{n}, \omega_{i}\left(t_{n}, x_{n}\right)\right)}\left\langle\left(\xi_{n},-p_{n}\right), \nu\right\rangle \leq 0, \quad \forall n \in \mathbb{N} .
$$

Given that $\Gamma_{i}$ is lower semicontinuous on $M_{i}$, for any $\nu \in \Gamma_{i}(t, x, z)$ there exists a sequence $\nu_{n} \rightarrow v$ with $\nu_{n} \in \Gamma_{i}\left(t_{n}, x_{n}, \omega_{i}\left(t_{n}, x_{n}\right)\right)$. Evaluating the last inequality at this sequence $\left\{v_{n}\right\}$, noticing that $\Gamma_{i}(t, x, z)=\Gamma_{i}\left(t, x, \omega_{i}(t, x)\right)$ and letting $n \rightarrow+\infty$ we obtain (3.10) for any $\eta \in \mathcal{N}_{\mathcal{E}_{i}}^{P}(t, x, z)$.

Now, consider $(t, x) \in \operatorname{dom} \vartheta, \tau \in(t, T]$ and $y \in \mathcal{S}_{t}^{\tau}(x)$ as in the statement.

Let $r>\tilde{r}>0$ large enough so that $y([t, \tau]) \subseteq \mathbb{B}_{\tilde{r}}$ and

$$
\sup _{X \in M \cap \mathbb{B}_{\tilde{r}}}\left|\operatorname{proj}_{\mathcal{E}_{i}}(X)\right|<r .
$$

Let $L_{i}$ be the Lipschitz constant for $\Gamma_{i}$ on $M_{i} \cap \mathbb{B}_{r}$. Notice that $\mathcal{E}_{i} \cap \bar{M}=\mathcal{E}_{i}$ and $X-\operatorname{proj}_{\mathcal{E}_{i}}(X) \in \mathcal{N}_{\mathcal{E}_{i}}^{P}(X)$ for any $X \in \mathcal{E}_{i}$. Therefore, it is not difficult to see that (3.10) implies (3.7) with $\kappa=L_{i}$. In particular, by Lemma 3.4 we have that for any absolutely continuous arc $\gamma:[t, \tau] \rightarrow \bar{M}_{i}$ which satisfies

$$
\dot{\gamma} \in \Gamma_{i}(\gamma) \quad \text { a.e. on }[t, \tau] \quad \text { and } \quad \gamma(s) \in M_{i} \cap \mathbb{B}_{r} \quad \forall s \in(t, \tau) \text {, }
$$

the following estimate holds:

$$
\operatorname{dist}_{\mathcal{E}_{i}}(\gamma(s)) \leq e^{L_{i} t} \operatorname{dist}_{\mathcal{E}_{i}}(\gamma(t)) \quad \forall s \in[t, \tau] .
$$

Finally, consider the absolutely continuous arc defined by

$$
\gamma_{y}(s)=(\tau+t-s, y(\tau+t-s), \omega(\tau, y(\tau))), \quad \forall s \in[t, \tau] .
$$

This curve fulfills the required conditions for (3.11) to hold, because $\dot{\gamma} \in \Gamma_{i}(\gamma)$ a.e. on $[t, \tau]$ and due to $y(s) \in \mathcal{M}_{i}$ for any $s \in(t, \tau)$, we have $\gamma_{y}(s) \in M_{i}$ for any $s \in(t, \tau)$.

Finally, since $\gamma_{y}(t)=(\tau, y(\tau), \omega(\tau, y(\tau))) \in \mathcal{E}_{i}$, (3.11) implies that $\gamma_{y}(\tau) \in \mathcal{E}_{i}$, which leads to $\omega(t, x)=\omega(t, y(t)) \leq \omega(\tau, y(\tau))$. This ends the proof of the claim.

Claim B: For any $(t, x) \in \operatorname{dom} \vartheta$ and $y \in \mathcal{S}_{t}^{T}(x)$ for which there exists a partition of $[t, T]$, $\left\{t=t_{0}<t_{1}<\ldots<t_{n}<t_{n+1}=T\right\}$, so that for any $l \in\{0, \ldots, n\}$ we can find $i \in \mathcal{I}$ such that $y(s) \in \mathcal{M}_{i}$ on $\left(t_{l}, t_{l+1}\right)$. Then, $\omega(t, x) \leq \omega(s, y(s))$ for any $s \in[t, T]$.

Proof. [Claim B] It is enough to apply on each subinterval $\left(t_{l}, t_{l+1}\right)$ Claim A.

With Claim B we have almost proved the desired result, because have shown that (3.8) implies that the strongly increasing principle for a class of regular trajectories. Finally, proof of the current proposition is accomplished in the light of Lemma 3.3 and reads as follows.

Let $(t, x) \in[0, T] \times \mathcal{K}, s \in[t, T]$ and $y \in \mathcal{S}_{t}^{T}(x)$, take a sequence $\left\{\varepsilon_{n}\right\} \subseteq(0,1)$ with $\varepsilon_{n} \rightarrow 0$. Let $x_{n} \in \mathcal{K}, t_{n} \in[0, T]$ and $y_{n} \in \mathcal{S}_{t_{n}}^{s}\left(x_{n}\right)$ given by Lemma 3.3 for $\varepsilon=\varepsilon_{n}$. By virtue of Claim $\mathrm{B}$, we have $\omega\left(t_{n}, x_{n}\right) \leq \omega(s, y(s))$. Therefore, due to $x_{n} \rightarrow x, t_{n} \rightarrow t$ and $\omega$ is l.s.c., the strongly increasing inequality holds and so the conclusion. 


\section{The minimal time problem}

The minimal time problem can be analyzed with the same arguments introduced for the Mayer problem of the last section. So here we merely state the main result and stress on the main differences.

Instead of a terminal cost function as in the Mayer problem, the minimal time problem has the additional data of a closed target set $\mathcal{C} \subseteq \mathbb{R}^{N}$. Parameterized by its initial point $x \in \mathbb{R}^{N}$, the problem is

$$
\min T-t \quad \text { over } y(\cdot) \text { satisfying }(\mathrm{DI})_{\mathcal{K}} \text { and } y(T) \in \mathcal{C},
$$

and denote by $\mathcal{T}(x)$ the optimal value in $(P(x))$. If no trajectory of (DI) originating from $x$ can reach $\mathcal{C}$ in finite time while staying in $\mathcal{K}$, then $\mathcal{T}(x)=+\infty$. Moreover, it is clear that $\mathcal{T}(x)=0$ whenever $x$ belongs to $\mathcal{C}$.

Proposition 4.1 For each $x \in \mathbb{R}^{N}$ where $\mathcal{T}(x)<\infty$, there exists an optimal solution to $(P(x))$. Moreover, $\mathcal{T}(\cdot)$ is l.s.c. on $\mathbb{R}^{N}$.

Proof. The unconstrained case is proven in [28, Proposition 2.6], and the proof is identical in the constrained case.

To state correctly the characterization of the minimum time function, we need to make precise the structure of the boundary of $\mathcal{C}$ and how it fits with the stratification of $\overline{\mathcal{K}}$. To make the exposure simple, we shall consider the case where the following holds:

$$
\text { There exists } \jmath \in \mathcal{I} \text { such that } \partial \mathcal{C} \subset \mathcal{M}_{\jmath} \text {. }
$$

Clearly, this hypothesis can be avoided but it is a simple case where the characterization of the minimum time function follows the same arguments as in the Mayer problem.

Theorem 4.2 Assume $\left(H_{F}\right),\left(H_{0}\right),\left(H_{1}\right)$ and $\left(H_{\mathcal{C}}\right)$. There exists a unique l.s.c. function $\Sigma(\cdot): \mathbb{R}^{N} \rightarrow$ $(-\infty,+\infty]$ that is bounded below on $\mathbb{R}^{N}$, satisfies the HJ equation

$$
\begin{aligned}
& -1+\sup _{v \in F^{\#}(x)}\{-\langle v, \zeta\rangle\} \geq 0 \quad \forall x \in \mathcal{K} \backslash \mathcal{C} \text { and } \zeta \in \partial_{P} \Sigma(x), \\
& -1+\sup _{v \in F_{i}(x)}\{-\langle v, \zeta\rangle\} \leq 0 \quad \forall x \in \mathcal{M}_{i} \backslash \mathcal{C} \text { and } \zeta \in \partial_{P} \Sigma_{i}(x),
\end{aligned}
$$

where $\Sigma_{i}(x)=\Sigma(x)+\chi_{\mathcal{M}_{i}}(x)$, and satisfies the boundary conditions

$$
\theta(x)=0 \quad \text { on } \mathcal{C}, \quad-1+\sup _{v \in F_{\jmath}(x)}\{-\langle v, \zeta\rangle\} \leq 0 \quad \forall \zeta \in \partial_{P} \Sigma_{\jmath}(x) \text { and } \forall x \in \partial \mathcal{C} .
$$

The unique such function is $\Sigma(\cdot)=\mathcal{T}(\cdot)$.

The proof of the above theorem uses the same arguments as the ones described in the previous section for the Mayer problem. The supersolution part is a classical result and does not need further attention. For the subsolution part, the main difference with the Mayer problem, is the behaviour of admissible trajectories that hit the strata $\mathcal{M}_{\mathcal{J}}$ (this special strata is involved in $\left(H_{\mathcal{C}}\right)$ ). Indeed, when an admissible trajectory hits $\mathcal{C}$, then it stops evolving and the condition (4.2) should be used to get the suboptimality principle for this trajectory on a small ball around the hitting time. Notice that the chattering behaviour cannot occur around $\partial \mathcal{C}$ since this boundary is absorbing (an admissible trajectory stops when it hits $\partial \mathcal{C})$. On an other hand, when an admissible trajectory hits $\mathcal{M}_{\mathfrak{J}} \backslash \mathcal{C}$, then the suboptimality principle comes from the condition (4.2), as for the Mayer problem. 


\section{Conclusion and comments}

Theorem 2.2 gives a characterization of the value function as the unique solution to a Hamilton-Jacobi equation on $\mathcal{K}$. This characterization or $\vartheta$ extends the known constrained viscosity solution to the case where $\mathcal{K}$ may have an empty interior.

The additional boundary conditions derived in this paper play a key role for the characterization of the l.s.c. value function $\vartheta$. Such boundary condition appear in the paper [11] in a more complicated form. Here by using the stratified structure of the state constraints set along with a local controllability assumption, the boundary condition takes a very simple and explicit form.

In the paper of Ishii-Koike [21], the case of discontinuous value function is studied without inward/outward condition. However, the set of constraints is assumed to have a nonempty interior, and it is assumed that at each point of the boundary, there exists an admissible trajectory lying in the set of constraints $\overline{\mathcal{K}}$. In this case, a notion of viscosity solution is defined by using the l.s.c. and u.s.c. enlevopes of the value function. In particular, the sub-optimality principle is expressed for the u.s.c envelope in terms of a HJ inequality using an Hamiltonian involving the dynamics pointing inside the set of constraints $\mathcal{K}$. Recall also that the result of Ishii-Koike insures the uniqueness of viscosity solution only when the inward condition is satisfied.

The uniqueness result holds whenever the assumptions $\left(H_{F}\right),\left(H_{1}\right)$ and $\left(H_{0}\right)$ are satisfied. It doesn't require the inward or outward condition to be satisfied. Also it is not necessary to have $F$ Lipschitz continuous every where. Only the Lipschitz continuity of each piece $F_{i}$ is required on the strata $\mathcal{M}_{i}$

It is now well established that the class of problems satisfying the "Inward Pointing Condition" (IPC) has very nice features [9]. However as far as the characterization of the value function is concerned, the IPC is not a necessary requirement. The sub-optimality and super optimality principles can be described very clearly by means of $\mathrm{HJ}$ equations when the set of constraints has a stratified structure and the dynamics satisfies $\left(H_{F}\right),\left(H_{1}\right)$.

Let us consider the simple example where $\overline{\mathcal{K}}:=[-1,1]^{2}$ is a square in $\mathbb{R}^{2}$ and consider the dynamics: $F(x)=\left(\max \left(\left|x_{1}\right|,\left|x_{2}\right|\right)^{2}-1\right) B(0 ; 1)+\left(\begin{array}{l}0 \\ 1\end{array}\right)$. In this simple example, one can see that neither the inward nor the outward condition is satisfied on all the boundary. Actually the IPC is satisfied only on $\{-1\} \times]-1,1$ [, the "Outward Pointing Condition" is satisfied on $\{1\} \times]-1,1[$, while on $]-1,1[\times\{ \pm 1\}$, the vector field is neither inward nor outward pointing. However, in this example, the condition $\left(H_{1}\right)$ is satisfied.

\section{Proof of the technical lemma}

As aforesaid, in this final part we provide a proof for Lemma 3.3. We recall that our proof relies heavily on the controllability assumption $\left(H_{2}\right)$. We recall that the statement of the lemma is as follows.

Lemma 3.3 Suppose $\left(H_{0}\right),\left(H_{1}\right)$ and $\left(H_{2}\right)$ hold. Let $(t, x) \in[0, T] \times \mathcal{K}$ and $y \in \mathcal{S}_{t}^{T}(x)$ be given, then for any $\varepsilon>0$ and $\tau \in[t, T]$ we can find $x_{\varepsilon} \in \mathbb{B}(x, \varepsilon) \cap \mathcal{K}, t_{\varepsilon} \in(t-\varepsilon, t+\varepsilon) \cap[0, \tau]$ and $y_{\varepsilon} \in \mathcal{S}_{t_{\varepsilon}}^{\tau}\left(x_{\varepsilon}\right)$ that verifies $y_{\varepsilon}(\tau)=y(\tau)$ and that is regular in the following sense:

There exists a partition of $[t, \tau],\left\{t=t_{0}<t_{1}<\ldots<t_{n}<t_{n+1}=\tau\right\}$, so that for any $l \in\{0, \ldots, n\}$ we can find $i \in \mathcal{I}$ such that $y_{\varepsilon}(s) \in \mathcal{M}_{i}$ on $\left(t_{l}, t_{l+1}\right)$

Proof. We exclusively do the case $\tau=T$, any other situation is analogous.

Let us assume there exists $i \in \mathcal{I}$ so that $J_{i}=\left\{s \in[t, T] \mid y(s) \in \mathcal{M}_{i}\right\}$ contains infinitely many disjoint open intervals, otherwise the triple $(x, t, y)$ satisfies the conclusion. Since the stratification is locally finite and the strata of $\mathcal{K}$ are disjoint, we might assume that $\mathcal{M}_{i}$ is unique and of minimal dimension; it may be, as the matter of fact, a finite union of strata of the same dimension. 
Because of the minimality of the dimension of $\mathcal{M}_{i}, J:=(t, T) \backslash J_{i}$ is open and, $a=\min J_{i}$ and $b=\max J_{i}$ are well-defined. So, for any $\varepsilon>0$ we can construct a partition of $[a, b]$

$$
b_{0}:=a \leq a_{1}<b_{1} \leq a_{2}<b_{2} \leq \ldots \leq a_{n}<b_{n} \leq b=: a_{n+1}
$$

that verifies

$$
\operatorname{meas}\left(J \backslash \bigcup_{l=1}^{n}\left(a_{l}, b_{l}\right)\right) \leq \varepsilon .
$$

with $y\left(a_{l}\right), y\left(b_{l}\right) \in J_{i}$ and $\left(a_{l}, b_{l}\right) \subseteq J$ for any $l=1, \ldots, n$. In addition,

$$
\bigcup_{l=0}^{n}\left[b_{l}, a_{l+1}\right] \backslash J_{i}=J \backslash \bigcup_{l=1}^{n}\left(a_{l}, b_{l}\right) .
$$

Hence, if we set $J^{l}:=\left[b_{l}, a_{l+1}\right] \backslash J_{i}$ and $\varepsilon_{l}=\operatorname{meas}\left(J^{l}\right)$, we have $\sum_{l=0}^{n} \varepsilon_{l} \leq \varepsilon$.

On the other hand, there must be some $l \in\{0, \ldots, n\}$ for which there is a countable family of intervals $\left(\alpha_{p}, \beta_{p}\right) \subseteq\left[b_{l}, a_{l+1}\right]$, pairwise disjoint that verifies $\varepsilon_{l}=\sum_{p \in \mathbb{N}}\left(\beta_{p}-\alpha_{p}\right), y(t) \in \mathcal{M}$ for any $t \in\left(\alpha_{p}, \beta_{p}\right)$ and $y\left(\alpha_{p}\right), y\left(\beta_{p}\right) \in \mathcal{M}_{i}$. Without loss of generality we might assume that each $l \in\{0, \ldots, n\}$ for which $b_{l}<a_{l+1}$, verifies this property.

Since there are infinitely many $\left\{\alpha_{p}\right\}$ and $[t, T]$ is compact, it has an accumulation point, say $\alpha \in[t, T]$. The same argument used in the necessity part of Proposition 3.2 allows us to show that any accumulation point of $v_{p}:=\frac{1}{\alpha_{p}-\alpha}\left(y\left(\alpha_{p}\right)-y(\alpha)\right)$ belongs to $F(y(\alpha))$. From where dom $F_{i} \neq \emptyset$.

Let $r>0$ so that $y(s) \in \mathbb{B}(0, r)$ for any $s \in[t, T]$. Consider as well $\varepsilon_{i}>0$ and $\Delta_{i}>0$ the constant given by $\left(H_{2}\right)$, and suppose $\varepsilon \leq \varepsilon_{i}$. So, for any $p \in \mathbb{N}$, if we set $\tau_{p}=\alpha_{p}+\Delta_{i}\left(\beta_{p}-\alpha_{p}\right)$, we can pick $y_{p} \in \mathcal{S}_{\alpha_{p}}^{\tau_{p}}\left(y\left(\alpha_{p}\right)\right)$ and $t_{p} \in\left(\alpha_{p}, \tau_{p}\right]$ such that

$$
y_{p}(s) \in \mathcal{M}_{i}, \forall s \in\left[\alpha_{p}, t_{p}\right), \quad y_{p}\left(\alpha_{p}\right)=y\left(\alpha_{p}\right), \quad \text { and } \quad y_{p}\left(t_{p}\right)=y\left(\beta_{p}\right) .
$$

Let $J_{i}^{l}:=\left[b_{l}, a_{l+1}\right] \cap J_{i}$ and the measurable function $\omega:\left[b_{l}, a_{l+1}\right] \rightarrow \mathbb{R}$

$$
\omega(s)=\mathbb{1}_{J_{i}^{l}}(s)+\sum_{p \in \mathbb{N}} \frac{t_{p}-\alpha_{p}}{\beta_{p}-\alpha_{p}} \mathbb{1}_{\left(\alpha_{p}, \beta_{p}\right)}(s)>0, \quad \forall s \in\left[b_{l}, a_{l+1}\right] .
$$

Accordingly, the map $s \mapsto \nu(s)=b_{l}+\int_{b_{l}}^{s} \omega(\tau) d \tau$ defined on $\left[b_{l}, a_{l+1}\right]$ is a homeomorphism from $\left[b_{l}, a_{l+1}\right]$ into $\left[b_{l}, c_{l+1}\right]$. Moreover, $\left(t_{p}-\alpha_{p}\right) \leq \Delta_{i}\left(\beta_{p}-\alpha_{p}\right)$ which leads to

$$
c_{l+1}-a_{l+1}=\operatorname{meas}\left(J_{i}^{l}\right)-\left(a_{l+1}-b_{l}\right)+\sum_{p \in \mathbb{N}}\left(t_{p}-\alpha_{p}\right) \leq \Delta_{i} \varepsilon_{l} .
$$

Consider the measurable function $v_{l}:\left[b_{l}, c_{l+1}\right] \rightarrow \mathbb{R}^{N}$ given by

$$
v_{l}(s)=\dot{y}\left(\nu^{-1}(s)\right) \mathbb{1}_{J_{i}^{l}}\left(\nu^{-1}(s)\right)+\sum_{p \in \mathbb{N}} \dot{y}_{p}(s) \mathbb{1}_{\left(\alpha_{p}, \beta_{p}\right)}\left(\nu^{-1}(s)\right), \quad \text { for a.e. } s \in\left[b_{l}, c_{l+1}\right] .
$$

Let $\tilde{y}_{l}:\left[b_{l}, c_{l+1}\right] \rightarrow \mathbb{R}^{N}$ be defined via

$$
y_{l}(s)=y\left(b_{l}\right)+\int_{b_{l}}^{s} v_{l}(\tau) d \tau, \quad \forall s \in\left[b_{l}, a_{l+1}\right] .
$$

By construction $y_{l}(\nu(t))=y(t)$ for any $t \in J_{i}^{l}$ and $y_{l}(t) \in \mathcal{M}_{i}$ for any $t \in\left[b_{l}, c_{l+1}\right]$. In particular, $y_{l}\left(c_{l+1}\right)=y\left(a_{l+1}\right)$.

If $l \in\{0, \ldots, n\}$ is so that $b_{l}=a_{l+1}$, we set $c_{l+1}=b_{l}$ and $y_{l}\left(c_{l+1}\right)=y\left(a_{l+1}\right)$.

Therefore, doing the same procedure for each $l \in\{0, \ldots, n\}$, we can construct inductively an absolutely continuous curve $y_{\varepsilon}$ in the following way: 
- Set first

$$
\begin{array}{lrl}
y_{\varepsilon}(s) & =y(s), \quad s \in\left[t, t_{0}\right], & t_{0}=b_{0} \\
y_{\varepsilon}(s)=y_{0}(s), \quad s \in\left[t_{0}, t_{1}\right], & t_{1}=c_{1} .
\end{array}
$$

- Then for any $l \in\{1, \ldots, n\}$

$$
\begin{array}{lrlrl}
y_{\varepsilon}(s) & =y\left(a_{l}-t_{2 l-1}+s\right), & s \in\left[t_{2 l-1}, t_{2 l}\right], & t_{2 l} & =t_{2 l-1}+b_{l}-a_{l} \\
y_{\varepsilon}(s) & =y_{l}\left(b_{l}-t_{2 l}+s\right), & s \in\left[t_{2 l}, t_{2 l+1}\right], & t_{2 l+1}=t_{2 l}+c_{l+1}-b_{l} .
\end{array}
$$

- Finally, $y_{\varepsilon}(s)=y\left(a_{n+1}-t_{2 n+1}+s\right)$ for $s \in\left[t_{2 n+1}, T_{\varepsilon}\right]$ with $T_{\varepsilon}=t_{2 n+1}+T-a_{n+1}$.

Notice that $\left[b_{l}, a_{l+1}\right]=J_{i}^{l} \cup J^{l}$, so $c_{l+1}-b_{l} \geq \operatorname{meas}\left(J_{i}^{l}\right)=a_{l+1}-b_{l}-\varepsilon_{l}$. Hence, after a few algebraic steps we obtain, by virtue of (6.1),

$$
T_{\varepsilon}=T+\sum_{l=0}^{n}\left(c_{l+1}-a_{l+1}\right) \in\left[T-\varepsilon, T+\Delta_{i} \varepsilon\right] .
$$

To summarize, we have constructed a trajectory of the control systems for which the set $\{s \in$ $\left.\left[t, T_{\varepsilon}\right] \mid y_{\varepsilon}(s) \in \mathcal{M}_{i}\right\}$ can be decomposed into a finite number of intervals. Furthermore, this trajectory verifies $y_{\varepsilon}(t)=x$ and $y_{\varepsilon}\left(T_{\varepsilon}\right)=y(T)$. Notice that process described above can also be applied to $y_{\varepsilon}$ but in this case the manifold that plays the role of $\mathcal{M}_{i}$ has dimension strictly larger than $\mathcal{M}_{i}$. We can then repeat procedure one more time for the resulting trajectory and once again the dimension of the manifold playing the role of $\mathcal{M}_{i}$ is strictly larger than the preceding one. Thus, it is clear that this scheme finishes in a finite number of step (there are only $N$ possible choices for the dimension of $\mathcal{M}_{i}$ ), and the resulting trajectory verifies the conditions of Claim B.

Since $\varepsilon>0$ is arbitrary and $\Delta_{i}>0$ does not depends upon $\varepsilon$, we may assume that $T_{\varepsilon} \in(T-\varepsilon, T+\varepsilon)$; using $\min \left\{\varepsilon, \frac{1}{\Delta_{i}} \varepsilon\right\}$ instead of $\varepsilon$ for instance.

Finally, re-scaling $\varepsilon$ if necessary, we can assume that $y_{\varepsilon}\left(t+T_{\varepsilon}-T\right) \in \mathbb{B}(x, \varepsilon)$. Therefore, to complete the proof it is enough to take $t_{\varepsilon}=T_{\varepsilon}$ and $x_{\varepsilon}=x$ if $T_{\varepsilon} \leq T$ or $t_{\varepsilon}=T$ and $x_{\varepsilon}=y_{\varepsilon}\left(t+T_{\varepsilon}-T\right)$.

\section{References}

[1] A. Altarovici, O. Bokanowski, and H. Zidani. A general Hamilton-Jacobi framework for nonlinear state-constrained control problems. ESAIM: Control, Optimisation and Calculus of Variations, 19(2): 337-357, 2013.

[2] A.V. Arutyunov and N.T. Tynyanskiy. The maximum principle in a problem with phase constraints. Soviet Journal of Computer and System Sciences, 23:28-35 (1985). [Translated into English. Original Russian text was published in 1984]

[3] A.V. Arutyunov. On Necessary Conditions for Optimality in a Problem with State Constraints. Dokl. Akad. Nauk SSSR, 280(5):1033-1037 (1985).

[4] A.V. Arutyunov. On the theory of the maximum principle in optimal control problems with phase constraints. Soviet Math. Dokl., 39(1): 1-4 (1989).

[5] J.P. Aubin and A. Cellina. Differential inclusions: set-valued maps and viability theory. Volume 264 of Grundlehren der mathematischen Wissenschaften. Springer-Verlag New York, 1984.

[6] M. Bardi and I. Capuzzo-Dolcetta. Optimal control and viscosity solutions of Hamilton-JacobiBellman equations. Systems \& Control: Foundations \& Applications. Birkhäuser Boston Inc., Boston, MA, 1997. With appendices by M. Falcone and P. Soravia. 
[7] R. C. Barnard and P. R. Wolenski. Flow invariance on stratified domains. Set-Valued and Variational Analysis, 21:377-403, 2013.

[8] E. N. Barron and R. Jensen. Semicontinuous viscosity solutions for Hamilton-Jacobi equations with convex Hamiltonians. Comm. Partial Differential Equations, 15(12):1713-1742, 1990.

[9] P. Bettiol, H. Frankowska, and R. B. Vinter. $l^{\infty}$ estimates on trajectories confined to a closed subset. Journal of Differential Equations, 252(2):1912-1933, 2012.

[10] A. Blanc. Deterministic exit time problems with discontinuous exit cost. SIAM J. Control Optim., 35:399-434, 1997.

[11] O. Bokanowski, N. Forcadel, and H. Zidani. Deterministic state-constrained optimal control problems without controllability assumptions. ESAIM: Control, Optimisation and Calculus of Variations, 17(4):995-1015, 2011.

[12] F. Clarke. Functional analysis, calculus of variations and optimal control. Volume 264 of Graduate Text in Mathematics. Springer, 2013.

[13] F. Clarke, Y. Ledyaev, R. Stern, and P. Wolenski. Nonsmooth Analysis and Control Theory. Volume 178 of Graduate Text in Mathematics. Springer, 1998.

[14] M. Crandall, L. Evans, And P.-L. Lions, Some properties of viscosity solutions of HamiltonJacobi equations, Trans. Amer. Math. Soc, 282:487-502 (1984).

[15] A.Y. Dubovitskii and V.A. Dubovitskii. Necessary conditions for strong minimum in optimal control problems with degeneration of endpoint and phase constraints. Usp. Mat. Nauk., 40(2): 175-176 (1985).

[16] N. Forcadel, Z. Rao, and H. Zidani. State-constrained optimal control problems of impulsive differential equations. Applied Mathematics \& Optimization, 68:1-19, 2013.

[17] H. Frankowska. Lower semicontinuous solutions of Hamilton-Jacobi-Bellman equations. SIAM Journal on Control and Optimization, 31(1):257-272, 1993.

[18] H. Frankowska and S. Plaskacz. Semicontinuous solutions of Hamilton-Jacobi-Bellman equations with degenerate state constraints. J. Math. Anal. Appl., 251(2):818-838, 2000.

[19] H. Frankowska and R. B. Vinter. Existence of neighboring feasible trajectories: Applications to dynamics programming for state-constrained optimal control problems. Journal of Optimization Theory and Applications, 104(1):20-40, 2000.

[20] C. Hermosilla and H. Zidani. Infinite horizon problems on stratifiable state constraints sets. J. Differential Equations 258 (4):1430-1460, 2015.

[21] H. Ishii and S. Koike. A new formulation of state constraint problems for first-order pdes. SIAM Journal on Control and Optimization, 34(2):554-571, 1996.

[22] J. Lee. Introduction to smooth manifolds. Volume 218 of Graduate Text in Mathematics. Springer, 2013

[23] M. Motta. On nonlinear optimal control problems with state constraints. SIAM J. Control Optim., 33:1411-1424, 1995.

[24] M. Motta and F. Rampazzo. Multivalued dynamics on a closed domain with absorbing boundary. applications to optimal control problems with integral constraints. Nonlinear Analysis, 41:631$647,2000$. 
[25] H. Soner. Optimal control with state-space constraint I. SIAM Journal on Control and Optimization, 24(3):552-561, 1986.

[26] H. Soner. Optimal control with state-space constraint II. SIAM Journal on Control and Optimization, 24(6):1110-1122, 1986.

[27] G. Smirnov. Introduction to the theory of differential inclusions. Volume 41 of Graduate Studies in Mathematics. AMS, 2002.

[28] P. Wolenski and Y. Zhuang. Proximal analysis and the minimal time function. SIAM J. Control Optim., 36(3):1048-1072 (electronic), 1998. 